\title{
Composition of Piperine with Enteric-Coated Chitosan Microspheres Enhances the Transepithelial Permeation of Curcumin in Sheep Intestinal Mucosa and Caco-2 Cells
}

\author{
Githa Thomas ${ }^{1}$ Marina Koland ${ }^{10}$ \\ ${ }^{1}$ Department of Pharmaceutics, NGSM Institute of Pharmaceutical \\ Sciences, Nitte (Deemed to be University), Mangalore, Karnataka, \\ India \\ J Health Allied Sci ${ }^{\mathrm{NU}}$ 2022;12:312-321.
}

\begin{abstract}
Address for correspondence Marina Koland, MPharm, PhD, Department of Pharmaceutics, NGSM Institute of Pharmaceutical Sciences, Nitte (Deemed to be University), Paneer, Deralakatte, Mangalore, Karnataka 575 018, India (e-mail: marinakol@nitte.edu.in).
\end{abstract}

\begin{abstract}
Objectives The purpose of this study was to investigate the efficacy of enteric-coated chitosan microspheres with herbal bioenhancer, piperine, as a suitable composition for improving the permeation of curcumin through biological membranes using suitable ex vivo models.

Material and Methods Chitosan microspheres of curcumin and piperine were prepared by an emulsion cross-linking method using glutaraldehydes the cross-linking agent and characterized for size, shape, entrapment efficiency, mucoadhesion, and in vitro release. The effect of piperine on the permeation of curcumin through excised sheep intestinal mucosa and Caco-2-cell monolayer was investigated.

Statistical Analysis The data from permeation studies were analyzed by Student's ttest using Statistical Package for the Social Sciences (SPSS) software (SPSS, Chicago, IL, United States) with p-values $<0.05$ indicating statistical significance.

Results The formulations showed mucoadhesion for a period of more than 6 hours which was influenced by the chitosan content. The rate of drug release of uncoated formulation followed first-order kinetics, and the mechanism of release was non-Fickian transport. Optimized formulation was coated with a pH-sensitive polymer, Eudragit S100 , by a solvent evaporation technique in different concentrations and evaluated for

Keywords

- bioenhancer

- curcumin

- chitosan

- enteric coating

- microspheres

- piperine ex vivo permeation through sheep intestinal mucosa and Caco-2-cell monolayer. Scanning electron microscopy images of the optimized coated formulation showed spherical particles with smooth surfaces. The calculated permeation flux and permeability coefficient of curcumin from microspheres were at least $20 \%$ greater in the presence of piperine through the intestinal mucosa and 30\% through the Caco-2-cell monolayer model. The permeability coefficient of curcumin from microspheres with piperine was $1.93 \times 10$ to $5 \mathrm{~cm} / \mathrm{sec}$ and significantly greater $(p<0.05)$ than that of microspheres devoid of piperine and from aqueous dispersion $(p<0.005)$.
\end{abstract}

published online January 18,2022
DOI https://doi.org/ $10.1055 / \mathrm{s}-0041-1741417$ ISSN 2582-4287.

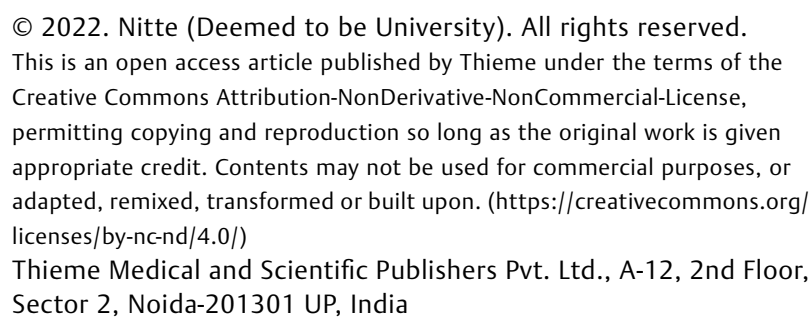


Conclusion The study confirmed the contribution of piperine and mucoadhesive microspheres toward improved permeation of curcumin through biological membranes, thereby providing an approach that has the potential of increasing transport through intestinal epithelial cells and possibly enhancing the oral bioavailability of this drug.

\section{Introduction}

In recent years, the importance of herbal-based drug delivery systems has increased tremendously. Herbal formulation is a dosage form in which phytoconstituents extracted from plants are incorporated for the treatment of various lifestyle diseases. The utilization of different herbal drugs like curcumin, silymarin, quercetin, neem, and so forth is expanding in our developing world because of the escalating cost of synthetic drugs, inadequate supply of drugs, as well as their side effects. ${ }^{1}$ World Health Organization estimated that more than $80 \%$ of the people worldwide depend on traditional medical practices for goals of primary health care. ${ }^{2}$ There are several reasons for the popularity of herbal medicines, chief among them being fewer side effects from natural medicines, a growing concern over the dependence and security of synthetic drugs and surgery, and failure of such drugs to successfully treat a significant number of common health conditions. $^{3}$

Curcumin is a polyphenolic constituent obtained from the rhizomes of Curcuma longa belonging to the family Zingiberaceae. Curcumin is known as a "cleanser of the body," in Ayurvedic medicine, and science has proved that there are many diseased conditions that can be cured by the active constituents of turmeric. Curcumin has antioxidant, antitumor, anti-inflammatory, antiviral, antibacterial, antifungal properties and, thus, has potential against different diseases including diabetes, asthma, allergies, arthritis, atherosclerosis, neurodegenerative diseases like Alzheimer's disease, and other chronic illnesses like cancer. ${ }^{4}$

Despite its promising therapeutic efficacy and great safety profile, the use of curcumin is associated with certain limitations such as poor oral bioavailability due to poor aqueous solubility and short half-life. Curcumin undergoes extensive first-pass metabolism and is eliminated rapidly. Thus, its retention time in circulation is very short. ${ }^{5}$

To overcome these limitations, various formulations and techniques have been investigated for the purpose of improving the absorption of curcumin over the past decades. Such strategies include the use of carrier systems such as polymeric nanoparticles, microspheres, microemulsions, nanocrystals, liposomes, and nanogels. ${ }^{6,7}$

There is no dearth of studies where various means of enhancing curcumin absorption have been reported. These include complexation of curcumin with phospholipids, use of polysorbate micelles, self-assembling nanoparticles, and lecithin nanoemulsions, to name a few. ${ }^{8-12}$

Another important approach is the use of bioenhancers, which may help to promote the absorption of curcumin when co-administered. Bioenhancers are molecules which do not possess activity of their own but promote and augment the absorption and bioavailability of drugs incorporated with them. ${ }^{13}$ When bioenhancers are used along with a drug, the dose of the latter can be reduced as well as the possible risk of side effects and drug resistance. Piperine, garlic, glycyrrhizin, quercetin, and naringin are a few of such compounds derived from plant sources that have the ability to increase the bioavailability when administered with other drugs by enhancing their membrane permeation properties. Piperine is a major component of black pepper (Piper nigrum) and has been shown to increase the oral bioavailability of curcumin by $2,000 \%$ after co-administration in humans at a weight ratio of $1: 200$, with no adverse effects in the doses used. ${ }^{14}$ The absorption enhancement properties of piperine are attributed to several mechanisms such as inhibition of drug-metabolizing enzymes, increase in gastro-intestinal (GI) blood supply, inhibition of drug efflux pump, or increase in membrane permeability due to epithelial cell modification. ${ }^{15-17}$

Microspheres are spherical and free-flowing particles ranging in average particle size from 1 to 1,000 microns which comprise proteins and synthetic polymers. Microspheres are often used as drug carriers for the purpose of controlled drug delivery as multiparticulate systems and are administered in capsules or tablets. These particles also help to enhance the absorption and stability of entrapped drugs by providing protection against the degrading effects of GI secretions and enzymes. Since the microspheres have a small size range, they may be able to easily penetrate through the absorbing membrane. ${ }^{18-20}$

Among the various polymers available for preparing microspheres, chitosan may be used when mucoadhesive properties are required. Chitosan is a polysaccharide obtained by n-deacetylation from chitin and has been widely used as a carrier for novel delivery systems owing to its biocompatibility and safety. Several studies have reported that chitosan can enhance the absorption of drugs into intestinal mucosa by mucoadhesion or by opening tight junctions between epithelial cells. ${ }^{21}$ Chitosan increases the penetration of the encapsulated drug through the mucosa. Besides mucoadhesion and absorption-enhancing properties, chitosan has other advantages like low toxicity, good biocompatibility, and antimicrobial effects. In our study, we have prepared chitosan microspheres entrapping a combination of curcumin and piperine, in an attempt to improve the absorption of curcumin through the GI mucosa.

Recently, there have been reports of the success of piperine in improving the intestinal transport of curcumin as 
nanoparticles, nanovesicles, or microspheres. ${ }^{22-24}$ There are also studies where the enteric coating of microspheres has been useful in the colon targeting of curcumin in the treatment of inflammatory bowel disease or for bioavailability enhancement. ${ }^{7,25}$ To the best of our knowledge, there are no reports of studies where a combination of enteric-coated chitosan microspheres and piperine as bioenhancer has been investigated in improving the intestinal transport of curcumin.

Since piperine is known to be associated with gastric irritation and as a result may cause nausea and vomiting, we have coated the chitosan microspheres with enteric polymers which prevents their disintegration or dissolution in gastric fluid but breaks down at a higher $\mathrm{pH}$ (in the small intestine). ${ }^{26}$ Enteric coating also prevents the premature adhesion and loss of the microspheres in the stomach instead of the intestine, which is the desired target site. Therefore, the present study is aimed at preparing and evaluating enteric-coated chitosan microsphere loaded with curcumin and piperine with improved intestinal permeation.

\section{Materials and Methods}

Curcumin and piperine were procured from Lobachemie, Mumbai, India, and Sami Labs Ltd., Bangalore, India, respectively. Chitosan, span 80 , and glacial acetic acid were obtained from Lobachemie, Mumbai. Eudragit S 100 was purchased from Evonik Pharma, Mumbai. All other chemicals and solvents were obtained from Merck Ltd, Mumbai.

\section{Preparation of Chitosan Microspheres}

Chitosan microspheres containing curcumin and piperine were prepared by the emulsion cross-linking method using glutaraldehyde as the cross-linking agent. Chitosan in different concentrations was dissolved in $5 \%(\mathrm{v} / \mathrm{v})$ of acetic acid. Curcumin and piperine in the ratio of 5:1 were dissolved in $5 \mathrm{~mL}$ methanol, and this solution was mixed with chitosan solution with stirring on a magnetic stirrer for 10 minutes at $500 \mathrm{rpm}$. The drug-polymer solution was then poured into $30 \mathrm{~mL}$ liquid paraffin containing $1 \%(\mathrm{w} / \mathrm{v}$ ) span 80 and stirred for 2 hours using a mechanical stirrer at $1,500 \mathrm{rpm}$. At the end of 2 hours, $1 \mathrm{~mL}$ of glutaraldehyde as the cross-linking agent was added to the emulsion and stirred for another 1 hour. Finally, the obtained microspheres were filtered, washed with n-hexane, and dried in desiccators for 24 hours. $^{27}$ The composition of different formulations is represented in - Table $\mathbf{1}$.

Table 1 Composition of different microsphere formulations

\begin{tabular}{|l|l|l|l|}
\hline $\begin{array}{l}\text { Formulation } \\
\text { code }\end{array}$ & $\begin{array}{l}\text { Curcumin } \\
\text { (mg) }\end{array}$ & $\begin{array}{l}\text { Piperine } \\
\text { (mg) }\end{array}$ & $\begin{array}{l}\text { Chitosan } \\
\text { (mg) }\end{array}$ \\
\hline F1 & 20 & 4 & 600 \\
\hline F2 & 20 & 4 & 800 \\
\hline F3 & 20 & 4 & 1,000 \\
\hline F4 & 20 & 4 & 1,200 \\
\hline F5 & 20 & 4 & 1,400 \\
\hline
\end{tabular}

\section{Evaluation of Uncoated Microspheres}

\section{Size and Shape Analysis}

Size analysis was performed by optical microscopy using the eyepiece and stage micrometer. A small quantity of microspheres was spread on a glass slide, and the particle size of 100 microspheres was measured randomly by optical microscopy. ${ }^{28}$ The size distribution and the average particle size of the microspheres were calculated by using the following equation:

$$
\text { Average size }=\frac{\Sigma n d}{\Sigma n}(1)
$$

where $n$ is the number of microspheres and $d$ is the diameter of the microsphere.

Furthermore, the size distribution was determined by digital imaging microscopy using the Biovis particle size analyzer (Primo Star, Zeiss) equipped with software for rapid analysis. This instrument makes the use of a high-power microscope fitted with a camera for taking digital images. The photomicrographs obtained were further analyzed by software to give size distribution.

\section{Scanning Electron Microscopy}

Scanning electron microscopy (SEM) was performed to determine the accurate shape and morphology of microspheres. Uncoated and coated microspheres of curcumin were examined. A small amount of microspheres was placed over a metal stub. Gold sputtering was performed for the metal stub and observed under SEM. ${ }^{29}$

\section{Entrapment Efficiency}

For this parameter, approximately $10 \mathrm{mg}$ of the prepared uncoated microspheres was crushed using a glass mortar and pestle. The powder obtained was extracted with $10 \mathrm{~mL}$ methanol in a Tarsus centrifuge tube and kept overnight. Then, the samples were centrifuged at $2,000 \mathrm{rpm}$ for 10 minutes at $4^{\circ} \mathrm{C}$. After centrifugation, the supernatant and the sediment were separated. The concentration of curcumin present in the supernatant was analyzed by the UV spectrophotometer at $421 \mathrm{~nm} .^{25}$ Entrapment efficiency (EE) was calculated by the following equation:

$$
\% \text { Entrapment efficiency }=\frac{\text { actual content }}{\text { theorectical content }} \times 100(2)
$$

\section{In Vitro Wash-Off Test for Mucoadhesion}

A freshly cut piece of sheep intestinal mucosa, collected within 1 hour after killing the animal, was mounted on a glass slide. A small quantity of uncoated microspheres was spread on the wet rinsed tissue on the mucosal side. The number of microspheres present on the surface of mucosa was counted under $45 x$ magnification. The slide was then hung from the horizontal bars of the USP Disintegration test apparatus so that it dipped into a $1 \mathrm{~L}$ capacity beaker containing $900 \mathrm{~mL}$ of phosphate buffer of $\mathrm{pH} 6.8$ maintained at $37 \pm 0.5^{\circ} \mathrm{C}$. The apparatus was operated so that the tissue moved up and down in the buffer. At predetermined time intervals, the glass slide was removed and the number of 
microspheres retained on the mucosa was counted under the microscope. The number of microspheres detached from the mucosa was obtained from the difference between the initial and final number counted. ${ }^{30}$ The percentage of mucoadhesion was calculated as per the following equation:

$\%$ Mucoadhesion $=\frac{\text { No.of microspheres adhered to the mucosa }}{\text { Total no.of microspheres spread on the mucosa }} \times 100(3)$

\section{In Vitro Drug Release Studies}

Drug release from the microspheres was performed using USP Dissolution Apparatus Type I in a medium of varying $\mathrm{pH}$ at $37 \pm 0.5^{\circ} \mathrm{C}$ with a rotation speed of $100 \mathrm{rpm}$. Chitosan microspheres corresponding to $20 \mathrm{mg}$ of curcumin were weighed and filled into a capsule and placed in the basket of the apparatus. The study was performed in $350 \mathrm{~mL}$ of $0.1 \mathrm{~N}$ $\mathrm{HCl}$ ( $\mathrm{pH} 1.2$ ) for the first 2 hours. At the end of the second hour, the $\mathrm{pH}$ was raised to 6.8 by adding $250 \mathrm{~mL}$ of a buffer prepared from $3.8 \mathrm{~g}$ of potassium dihydrogen phosphate and $2.27 \mathrm{~g}$ of sodium hydroxide that has been equilibrated to $37 \pm 0.5^{\circ} \mathrm{C}$, and the release study was continued for another 2 hours. At the end of the fourth hour, the $\mathrm{pH}$ was again changed to 7.4 by adding $300 \mathrm{~mL}$ of buffer containing $1.24 \mathrm{~g}$ of potassium dihydrogen phosphate and $0.54 \mathrm{~g}$ of sodium hydroxide and, further, the study was continued for an additional 4 hours in the case of uncoated microspheres and 8 hours for enteric-coated microspheres. Samples of $5 \mathrm{~mL}$ were withdrawn from the dissolution medium at suitable time intervals and replaced with fresh medium and analyzed by a UV spectrophotometer at $421 \mathrm{~nm}^{31}$

\section{Kinetic Analysis of Drug Release Data}

The drug release data were subjected to kinetic analysis using the software, PCP Disso v3 to ascertain the order of kinetics. Moreover, the data were also fitted to Higuchi matrix and Korsmeyer-Peppas models to determine the mechanism of release. $^{32}$

\section{Coating of Chitosan Microspheres}

Chitosan microspheres were coated with Eudragit S 100 by the solvent evaporation method. Eudragit S 100 was dissolved in $10 \mathrm{~mL}$ ethanol to prepare coating solutions of three different concentrations $(0.5,1.0$, and $1.5 \% \mathrm{w} / \mathrm{v})$. The chitosan microspheres $(50 \mathrm{mg}$ ) were dispersed in each of these solutions. Each of these dispersions was then incorporated into $50 \mathrm{~mL}$ liquid paraffin containing $1 \% \mathrm{w} / \mathrm{v}$ span 80 and stirred for 2 hours with a mechanical stirrer at $1,500 \mathrm{rpm}$. The coated microspheres were then collected, filtered, washed with nhexane, and dried in desiccators. ${ }^{25}$

The coating of the prepared microspheres was confirmed by a dye test. A pinch of crystal violet was added to the solution of Eudragit S 100 dissolved in ethanol. To this solution, a weighed amount of microspheres were added and the coating was done as per the procedure mentioned above. The obtained microspheres were then observed under optical microscopy (45x magnification) to confirm the presence of coating of the microspheres.
Evaluation of Enteric-Coated Chitosan Microspheres

The enteric-coated microspheres were characterized for size, shape, and in vitro release as per the methodology described earlier.

\section{In Vitro Swelling Studies}

The swelling index was determined by placing a pre-weighed dialysis bag (Himedia, molecular weight cut off: $12,000-$ 14,000 Daltons; pore size: $2.4 \mathrm{~nm}$ ) containing $100 \mathrm{mg}$ of microspheres in $200 \mathrm{~mL}$ of $0.1 \mathrm{~N} \mathrm{HCl}(\mathrm{pH} 1.2)$ and allowed to swell. At definite time intervals, the swollen microspheres were removed from the buffer at predetermined intervals, blotted with a filter paper for 10 seconds to remove the excess moisture and reweighed. The change in weight was determined until equilibrium was attained. ${ }^{25}$ The swelling index was calculated by the following formula:

Swelling index $=\frac{\text { Weight of swollen microsphere-initial weight }}{\text { initial weight }}$

The study was repeated in a phosphate buffer of $\mathrm{pH} 6.8$ and 7.4 for both coated and uncoated microspheres, respectively. The swelling profiles were obtained by plotting the swelling index against the time point of sampling in each case.

\section{Ex Vivo Drug Permeation Study through Sheep Intestinal Mucosa}

The ability of piperine to enhance the permeation of curcumin released from the selected formulation of enteric-coated microspheres was studied using a Franz diffusion cell with excised sheep intestinal mucosa as the model membrane. The intestinal mucosa which was previously equilibrated with phosphate buffer ( $\mathrm{pH}$ 7.4) was clamped between the donor and receptor compartments of the diffusion cell. Microspheres equivalent to $10 \mathrm{mg}$ of curcumin were gently spread on the mucosal surface that was facing the donor cell. About $5 \mathrm{~mL}$ of phosphate buffer of $\mathrm{pH} 6.8$ (intestinal $\mathrm{pH}$ ) was placed in the donor cell. The receptor compartment was filled with $25 \mathrm{~mL}$ of phosphate buffer of $\mathrm{pH} 7.4$ (physiological $\mathrm{pH}$ ) that was stirred at a constant speed of $100 \mathrm{rpm}$ and maintained at $37 \pm 0.5^{\circ} \mathrm{C}$. The study was performed for 6 hours. Samples were withdrawn from the receptor compartment at predetermined time intervals and replaced with fresh buffer to maintain sink conditions. ${ }^{33}$ The absorbance was measured using a UV spectrophotometer.

From the data of ex vivo permeation studies, the permeation flux and permeability coefficient were determined using the software, PCP Disso v3, based on the surface area of the membrane used and the time required for the study. ${ }^{34}$

\section{Ex Vivo Drug Permeability Study Using the Caco-2-Cell Monolayer Model}

Permeation of curcumin from the optimized microsphere formulation through the Caco-2-cell monolayer model in the presence of piperine was investigated.

Caco-2 cells were cultured in Dulbecco's modified eagle medium (DMEM) supplemented with $1 \%$ penicillin-streptomycin, 2 mM L-glutamine, $1 \%$ non-essential amino acids, and 
$10 \%$ fetal bovine serum at $37^{\circ} \mathrm{C}$ in an atmosphere of $5 \%$ carbon dioxide/95\% air/90\% RH. Using trypsin-ethylenediaminetetraacetic acid, cells were sub-cultured once in a week and seeded at the density of $4 \times 10$ cells $/ 75 \mathrm{~cm}^{2}$ flask in which the medium was changed daily.

The cultured cells were seeded on 6-well plates (Transwell) in the DMEM medium at a density of 10,000 cells/well. The integrity of the Caco-2-cell monolayer was checked by evaluating the cell polarization obtained by measuring the transepithelial electrical resistance before as well as at the end of the experiment. After 3 weeks, when adequate cell differentiation has occurred and tight monolayers have developed, samples of $100 \mu \mathrm{L}$ of curcumin dispersion $(1.5$ $\mathrm{mg} / \mathrm{mL}$ ) in DMEM or $10 \mathrm{mg}$ each of F3-B and F3-D equivalent to $150 \mu \mathrm{g}$ of curcumin were introduced into the apical chamber of the wells. Before application, the microspheres were gently agitated with $2 \mathrm{~mL}$ of phosphate buffer ( $\mathrm{pH} 6.8$ ) for 5 minutes to dissolve the enteric coating. Then, the permeation study was performed for 6 hours at $37^{\circ} \mathrm{C}$. At appropriate time intervals, samples were collected from the basolateral chamber of each well and analyzed using highperformance liquid chromatography. At the end of the study, the supernatant was separated and the cells were washed with phosphate buffer saline ( $\mathrm{pH} 7.4$ ). Then, the cells were scraped out from the wells to centrifuge tubes and subjected to centrifugation at $1,200 \mathrm{rpm}$ at $4^{\circ} \mathrm{C}$ for 5 minutes. The sediment of cells was extracted with methanol by vortexing to determine the accumulated curcumin in the Caco- 2 cells. The total amount of curcumin that has permeated across the monolayer corresponding to each time interval from each preparation was calculated. ${ }^{35}$ The permeation flux and permeability coefficient in each well was also determined.

The data were analyzed by Student's $t$-test using Statistical Package for the Social Sciences (SPSS) software (SPSS, Chicago, IL, USA). $p$-Values $<0.05$ indicated statistical significance.

\section{Results and Discussion}

Chitosan microspheres of curcumin were prepared by the emulsion cross-linking method wherein the mucoadhesive polymer was used in different concentrations keeping the amount of the drug and piperine constant. It was observed that the microspheres tended to be coagulated when chito- san exceeded the maximum concentrations indicated in -Table 1.

The average particle size of the uncoated chitosan microspheres determined by optical microscopy using the stage micrometer was found to be in the range of 12.99 to $23.21 \mu \mathrm{m}$. From the results, it was found that, as the concentration of the polymer increases, particle size also increased. From the digital imaging microscopy, the particle size of uncoated microspheres was found to be in the range of 0.5 to 25 microns. This instrument was able to detect particles less than 70 microns in size which could not be measured using an eyepiece micrometer. The values of particle size analysis are illustrated in -Table $\mathbf{2}$. The small particle size of the microspheres is important for better retention on the GI mucosa and permeation.

The shape and morphology of the microspheres were examined using SEM, and the images obtained are shown in - Fig. $\mathbf{1}(\mathbf{A})$. The uncoated chitosan microspheres are found to be spherical in shape with slightly rough surfaces, probably due to the hardening of the microspheres as a result of cross-linking by glutaraldehyde.

Results for EE of all uncoated formulations are shown in -Table 2. The uncoated microspheres demonstrated an increase in the EE with an increase in chitosan concentration up to a maximum of $97 \% \mathrm{w} / \mathrm{w}$. Hence, $\mathrm{F} 3$ showed a maximum EE of $75.26 \%$. Further increase in chitosan concentration did not make a difference to this parameter. Obviously, a greater polymer content ensures that the drug is completely entrapped within the matrix, and there is no leeway for escape.

The in vitro wash-off test was performed to study the mucoadhesive property of the uncoated microspheres, and the results are given in -Table 2. Percentage of

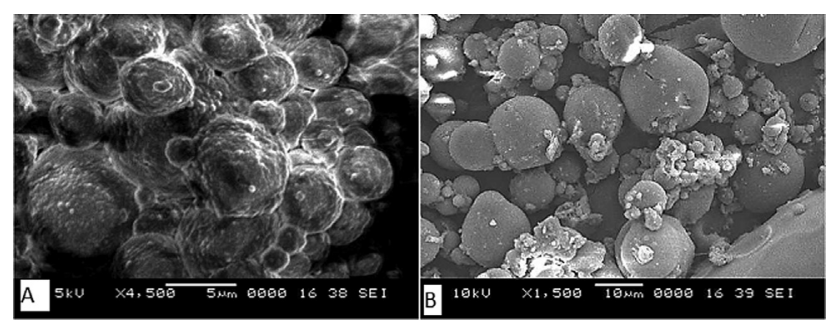

Fig. 1 SEM images of (A) uncoated chitosan microspheres, F3 and (B) coated microspheres, F3-Bof curcumin and piperine.

Table 2 Data for average particle size, entrapment efficiency, and percentage of mucoadhesion of uncoated chitosan microspheres

\begin{tabular}{|l|l|l|l|}
\hline $\begin{array}{l}\text { Formulation } \\
\text { code }\end{array}$ & $\begin{array}{l}\text { Average particle } \\
\text { size }(\mu \mathrm{m})^{\mathrm{a}}\end{array}$ & $\begin{array}{l}\text { Entrapment } \\
\text { efficiency }(\%)^{\mathrm{a}}\end{array}$ & $\begin{array}{l}\text { Percentage of mucoadhesion } \\
\text { at the end of }^{\mathrm{a}} \text { hours }^{\mathrm{a}}\end{array}$ \\
\hline F1 & $12.99 \pm 0.14$ & $42.56 \pm 2.23$ & $64.37 \pm 2.81$ \\
\hline F2 & $14.22 \pm 0.10$ & $56.46 \pm 1.14$ & $68.84 \pm 1.96$ \\
\hline F3 & $17.27 \pm 0.21$ & $75.26 \pm 1.72$ & $70.31 \pm 2.53$ \\
\hline F4 & $19.61 \pm 0.11$ & $73.41 \pm 2.05$ & $77.34 \pm 2.75$ \\
\hline F5 & $23.21 \pm 0.13$ & $70.44 \pm 1.77$ & $81.25 \pm 3.44$ \\
\hline
\end{tabular}

${ }^{\mathrm{a} E a c h}$ value is the average of three measurements with standard deviation. 


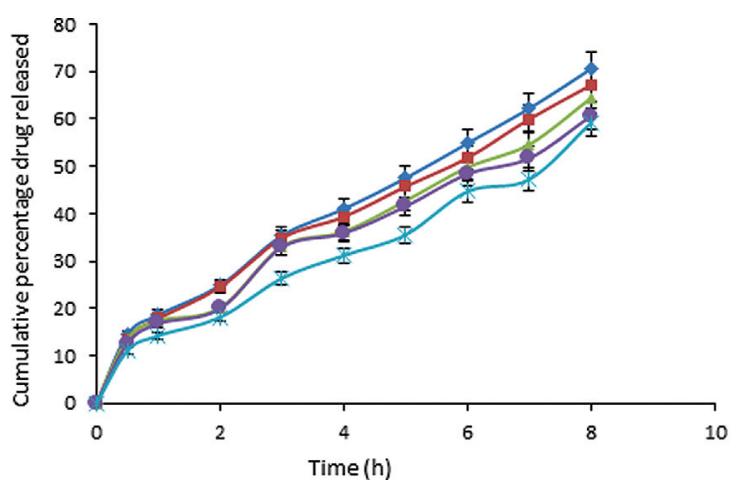

Fig. 2 Drug release profile from uncoated microsphere formulations at varying $\mathrm{GI} \mathrm{pH}$ ( 2 hours at pH 1.2, 2 hours at pH 6.8, and 4 hours at $\mathrm{pH}$ 7.4). Each value is the average of three determinations with standard deviation.

mucoadhesion recorded at the end of 6 hours for the formulations was found to increase with the chitosan content. Studies suggest that the mucoadhesive nature of chitosan is due to its cationic amine groups that can interact with the negatively charged mucosal glycoproteins. ${ }^{36,37}$ Thus, F5 showed the greatest mucoadhesion of $81.25 \%$ at the end of 6 hours.

The in vitro drug release profiles of uncoated chitosan microspheres are shown in - Fig. $\mathbf{2}$. The maximum percentage of drug release from the formulations ranged from 59.17 to $70.61 \%$. It was observed that the extent of drug release decreased with an increase in the chitosan content in the composition. In the first 2 hours, approximately 18 to $25 \%$ of curcumin was released, which means that we could expect the similar release in the stomach, if the microspheres were not enteric coated. This release may be due to the solubilization of chitosan in acidic pH. Studies have reported that drug release from chitosan microspheres can be due to three different mechanisms such as release from the particle surface, molecular diffusion through the swollen polymer matrix, and polymer erosion. ${ }^{38}$ Rapid drug dissolution from the particle surface is responsible for burst release. As the figure shows, there appears to be a burst release from all formulations in the first 30 minutes of the study. Further release would be more sustained since the swollen polymer matrix would produce a diffusion-controlled drug release. The release rate was also influenced by particle size which, in turn, depended upon polymer concentration. ${ }^{39}$ Thus, the release at the end of 8 hours was the lowest (59.2\%) from
F5 which had the largest microsphere size and the highest chitosan concentration.

The data obtained from the in vitro drug release study were fitted to different mathematical models such as zero order, first order, Higuchi, and Korsmeyer-Peppas to predict the kinetics and release mechanism, and the regression coefficient values obtained are represented in -Table 2 . Drug release followed first-order kinetics based on their higher regression coefficient values as compared with those of the zero-order model. This is expected of the matrix diffusion model since microspheres are micromatixes. The Peppas model was used to predict the mechanism of drug release from microspheres. The release exponent (n) of the Korsmeyer-Peppas equation for all formulations was found to be between 0.45 and 0.89 , thus indicating non-Fickian transport which is attributed to a combined effect of diffusion and erosion. ${ }^{40}$ Since the microspheres are micromatrixes, the mechanism of drug release could also be described by the Higuchi matrix diffusion principle. ${ }^{32}$ The regression coefficient values calculated for this model were also found to be significant as shown in - Table 3 .

On the basis of the data obtained from the characterization of the microspheres for particle size, $\mathrm{EE}$, and in vitro curcumin release, $\mathrm{F} 3$ was considered as optimal formulation and was selected for enteric coating and further investigation. When selecting this formulation, a suitable compromise was required between mucoadhesion and $\mathrm{EE}$, since the latter was more important than the former.

The enteric coating of the chitosan microspheres loaded with curcumin and piperine was essential to reduce the gastric irritation potential of the latter. Moreover, the coating also ensures that maximum drug concentrations would reach the intestine which is the major site of drug absorption. Once the coating disintegrates at the intestinal $\mathrm{pH}$, the mucoadhesiveness of chitosan ensures particle retention on the GI mucosa for better permeation.

The coating of the microspheres was done by the solvent evaporation technique using Eudragit S 100 in three ratios in three different coating concentrations of $0.5,1.0$, and $1.5 \%$ $(\mathrm{w} / \mathrm{v})$ to produce formulations $\mathrm{F} 3-\mathrm{A}, \mathrm{F} 3-\mathrm{B}$, and $\mathrm{F} 3-\mathrm{C}$ respectively. As the concentration of the polymer increases, the thickness of the coating layer is also increased. Microspheres were found to be agglomerated for coating ratios above 1:3.

Coating of the microspheres was confirmed by the dye test. The results showed that the coating of formulation F3-B was adequate although there was some sort of agglomeration

Table 3 Data for kinetic analysis of in vitro drug release data

\begin{tabular}{|l|l|l|l|l|l|l|}
\hline \multicolumn{2}{|l|}{ Release models } & FI & F2 & F3 & F4 & F5 \\
\hline Zero order & $R^{2}$ & 0.964 & 0.961 & 0.962 & 0.955 & 0.974 \\
\hline First order & $R^{2}$ & 0.989 & 0.990 & 0.986 & 0.986 & 0.981 \\
\hline Higuchi matrix & $R^{2}$ & 0.982 & 0.984 & 0.978 & 0.983 & 0.967 \\
\hline Korsmeyer-Peppas model & $R^{2}$ & 0.989 & 0.992 & 0.984 & 0.984 & 0.981 \\
\cline { 2 - 7 } & $n$ & 0.578 & 0.580 & 0.568 & 0.573 & 0.597 \\
\hline
\end{tabular}




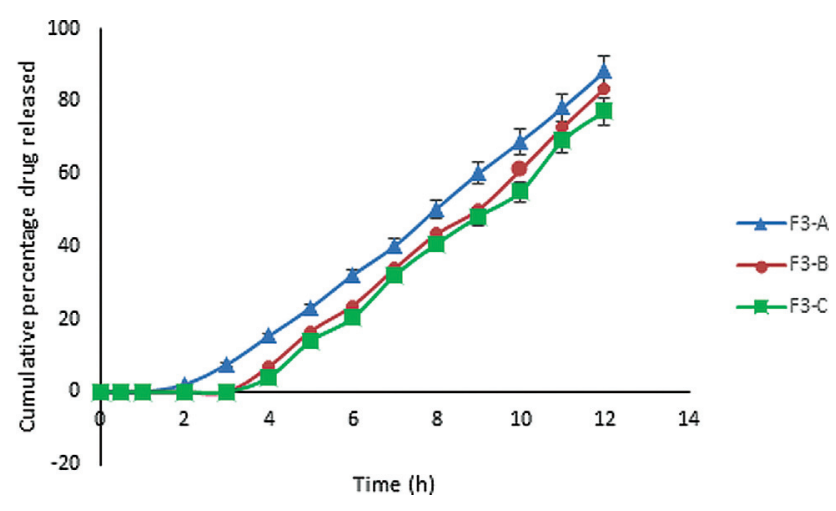

Fig. 3 Drug release profile from enteric-coated microspheres at varying $\mathrm{Gl} \mathrm{pH}$ ( 2 hours at pH 1.2, 2 hours at pH 6.8, and 8 hours at $\mathrm{pH}$ 7.4). Each value is the average of three determinations with standard deviation.

when compared with F3-A which was not perfectly coated and F3-B which have more agglomeration.

Coating of the chitosan microspheres with Eudragit S 100 showed a significant increase in the particle size. Digital imaging microscopy showed an average particle size of 63.53 to $72.76 \mu \mathrm{m}$. Increasing concentrations of the coating polymer resulted in the increase in the particle size. However, as the thickness of the coating increased, the incidence of agglomeration also increased. The formulation F3-C showed more agglomeration when compared with F3-A and F3-B.

From the SEM images of the coated microspheres as shown in -Fig. 1(B), it is observed that they retained their spherical shape with smoother surfaces, thus indicating uniformity of the coating. There were no signs of agglomeration.

The in vitro drug release profiles from the enteric-coated microspheres for a 12 hour study showed a progressive decrease in drug release with an increase in coating polymer composition as shown in -Fig. 3. There was no detectable drug release from $\mathrm{F} 3-\mathrm{B}$ and $\mathrm{F} 3-\mathrm{C}$ in the simulated gastric fluid of $\mathrm{pH} 1.2$ for up to 3 hours. On the contrary, there appeared to be some release from F3-A in the second hour which steadily increased thereafter to a maximum of $88.28 \%$ at the end of 12 hours. This observation indicates that the coating was probably defective and failed to prevent release at gastric $\mathrm{pH}$. In case of F3-B and F3-C, drug release could be detected in the fourth hour in the simulated intestinal fluid of $\mathrm{pH}$ 6.8. When the $\mathrm{pH}$ was changed to 7.4 , there was a dramatic increase in the release from all formulations, indicating the complete dissolution of the enteric coating. Thereafter, the curcumin release was sustained till the end of the study at 12 hours by diffusion through the swollen chitosan matrix and eventually its erosion.

The formulation, F3-B was selected for further studies, due to the defective coating of F3-A and the tendency of agglomeration in the case of $\mathrm{F} 3-\mathrm{C}$.

Swelling of a hydrogel polymer such as chitosan is an important factor that can influence its mucoadhesive properties. ${ }^{41}$ The swelling behavior of the polymer in the microspheres is also important for controlling the release of the drug. In a non-cross-linked polymer, too much swelling can ultimately result in the dissolution of chitosan and rapid drug release. In our study, we have cross-linked chitosan with glutaraldehyde which prevents the dissolution of the polymer, while it swells during imbibition of water and provides for a sustained release of curcumin. ${ }^{42}$ This study was performed for the optimized uncoated microsphere formulation F3 and the optimized enteric-coated counterpart, F3-B. The extent of swelling of the coated and uncoated microspheres from the water uptake or hydration was determined, and the swelling behavior at three different $\mathrm{pH}$ normally encountered during the passage of the microspheres in the gastrointestinal tract was compared. The results for in vitro swelling study of F3 and F3-B in pH 1.2, 6.8, and 7.4 are displayed as comparative profiles in - Fig. 4 (A, B, and C respectively). According to a study reported by Rohindra et al, the degree of swelling of chitosan hydrogels was the maximum at acidic $\mathrm{pH}$ and decreased with increasing $\mathrm{pH}^{43}$ This difference was attributed to the protonation of the amino groups of chitosan at low $\mathrm{pH}$ which led to increased water uptake but de-protonation at higher values of $\mathrm{pH}$ and subsequent shrinking of the gel network with reduced water uptake. Comparison of the swelling profiles of F3 and F3-B at pH 1.2 and 6.8, respectively, shows that there was indeed a greater degree of swelling of the uncoated microspheres in acidic $\mathrm{pH}$ but markedly reduced at the higher $\mathrm{pH}$. However, the opposite was the case with F3-B, since the enteric coating prevented the acid medium in which the microspheres were immersed from reaching the chitosan surface but dissolved sufficiently at the alkaline pH to permit access. The lower swelling index of coated microspheres ensures their resistance to swelling in the acidic contents in the stomach, thereby preventing premature release at a non-target site. At $\mathrm{pH} 7.4$, there was a noticeable decrease in swelling of F3 as predicted and not very different from F3-B. The slightly greater degree of swelling of the latter at $\mathrm{pH} 7.4$ when compared with $\mathrm{pH} 6.8$ could be due to the complete

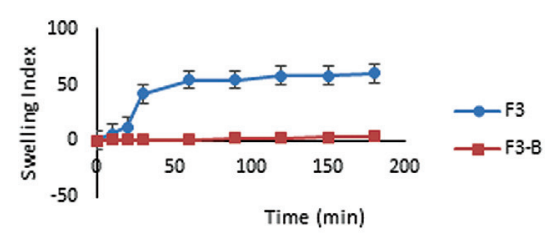

A

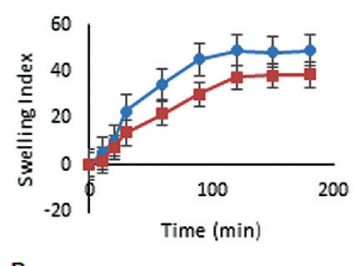

B
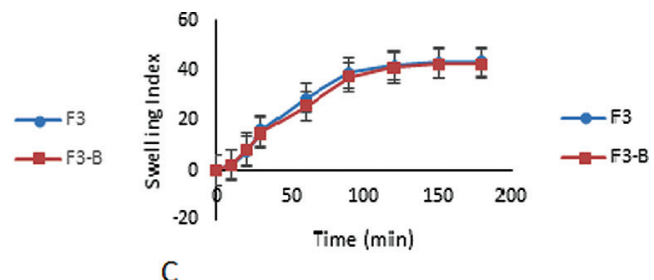

Fig. 4 In vitro swelling profile of (A) F3 and F3-B in $0.1 \mathrm{~N} \mathrm{HCl}$, (B) phosphate buffer of $\mathrm{pH} 6.8$ and (C) phosphate buffer of $\mathrm{pH} 7.4$. Each value is the average of three determinations with standard deviation. 
dissolution of the enteric coating allowing free access of the medium to the polymer matrix surface.

An ex vivo permeation study was performed using sheep intestinal mucosa to determine the effect of piperine on the permeation of curcumin through the intestinal mucosa. For this purpose, enteric-coated microspheres were prepared similar in composition to F3-B but with the exclusion of piperine and the formulation was coded as F3-D. Curcumin permeation through sheep intestinal mucosa from F3-B was compared with F3-D to understand the impact of piperine. It can be observed in - Fig. $\mathbf{5}$ that in the first $\mathbf{3 0}$ minutes, there was a time lag or delay in drug permeation because the enteric coating needed to dissolve before curcumin could be released. It was observed subsequently that in the first hour, there was an initial jump in the percentage of drug permeated from both formulations which coincided with the burst release observed in the in vitro release profile. This was attributed to the release of curcumin from the saturated outer layer of the microspheres. The cumulative percentage of drug permeated at the end of 6 hours from $\mathrm{F} 3-\mathrm{B}$ was found to be $57.77 \%$ and that from F3-D was $46.92 \%$. Thus, there was a $10 \%$ increase in the release of curcumin from the microspheres in the presence of piperine. This indicates that the absorption of curcumin was enhanced in the presence of piperine.

Using regression analysis, the slope of the linear portion of the plot was calculated and divided by the mucosal surface area to give the permeation flux. The steady-state permeability coefficient was calculated by dividing the permeation flux by the donor-phase drug concentration. ${ }^{44}$ The permeation flux from F3-B and F3-D were 1.2 and $0.976 \mathrm{mg} / \mathrm{cm}^{2} / \mathrm{h}$, while the permeability coefficients were $1.672 \times 10^{-5}$ and $1.356 \times 10^{-5} \mathrm{~cm} / \mathrm{sec}$ respectively. These results indicate that the permeation of curcumin through the intestinal mucosa was substantially increased in the presence of piperine.

When cultured under specific conditions, the Caco-2-cell monolayer can be considered as a model of the intestinal membrane. Carefully cultured Caco-2 cells are capable of spontaneous proliferation and differentiation and have typical characteristics of intestinal epithelium such as brushborder microvilli, tight intercellular junctions, and even possess various metabolic enzymes. In addition, this layer can act as a physical and biochemical barrier to the perme-

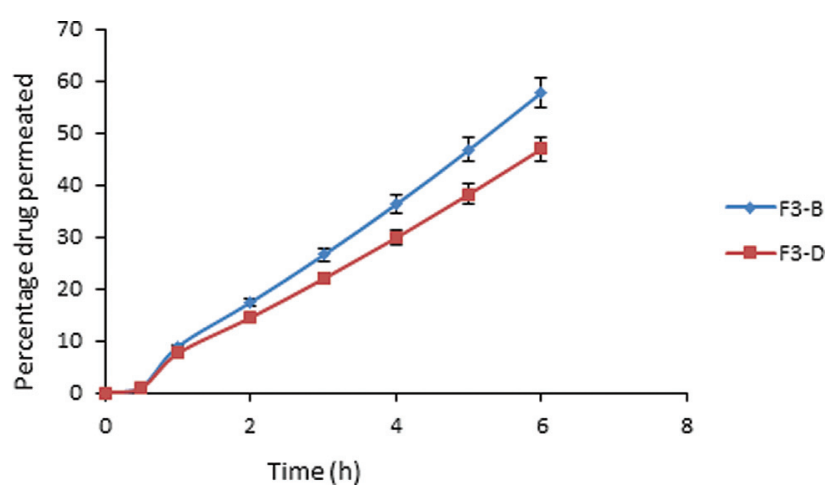

Fig. 5 Ex vivo permeation profiles of curcumin from F3-B and F3-D through sheep intestinal mucosa. Each value is the average of three determinations with standard deviation. ation of small molecules and ions as is the case with the intestinal mucosa. Therefore, permeation studies using Caco2 cells can provide valuable information regarding the intestinal absorption of drugs. Drug permeation through the Caco-2 cell monolayer for F3-B, F3-D and solution was determined and compared. The permeation profiles of curcumin are given in - Fig. $\mathbf{6}$. The percentage of drug permeated through Caco- 2 cells at the end of 6 hours was found to be $60.26 \%$ from $\mathrm{F} 3-\mathrm{B}, 42.17 \%$ from $\mathrm{F} 3-\mathrm{D}$, and $5.23 \%$ from the curcumin aqueous dispersion. The calculated values of permeation flux from F3-B, F3-D, and dispersion were 10.43, 7.66 , and $0.675 \mu \mathrm{g} / \mathrm{cm}^{2} / \mathrm{h}$, while the permeability coefficients were $1.93 \times 10^{-5}, 1.42 \times 10^{-5}$, and $1.25 \times 10^{-6} \mathrm{~cm} / \mathrm{sec}$, respectively. Thus, the presence of piperine produced a $30 \%$ increase in curcumin permeation from $\mathrm{F} 3-\mathrm{B}$ as compared with F3-D. The permeability coefficient of curcumin from F3B was significantly greater $(p<0.05)$ than F3-D or the dispersion $(p<0.005)$. As was expected, the permeation from the aqueous dispersion was almost negligible, since a large portion of the curcumin was in the undissolved form. These results further confirmed the fact that piperine increased the permeation of curcumin through Caco-2 cells which ensures better absorption and bioavailability. Further, it was established that the administration of curcumin as microspheres as compared with the simple aqueous dispersion made a significant contribution $(p<0.05)$ to the absorption of curcumin. This observation was credited to the mucoadhesive mature of the chitosan particles that provided intimate contact to the cell monolayer, in addition to providing sustained release which would have given sufficient time for the released drug to be absorbed.

In conclusion, enteric-coated chitosan microspheres of curcumin and piperine were prepared successfully by using the emulsion cross-linking method with glutaraldehyde as the cross-linker. The prepared microspheres showed good encapsulation efficiency, mucoadhesive property, swelling degree, and in vitro drug release. The enteric coating of the microspheres protects the stomach lining from the irritant effects of the piperine while ensuring undiluted concentrations of curcumin reach the site of absorption in the intestine. Ex vivo permeation of curcumin from the optimized microspheres through the sheep intestinal mucosa and Caco-2-cell

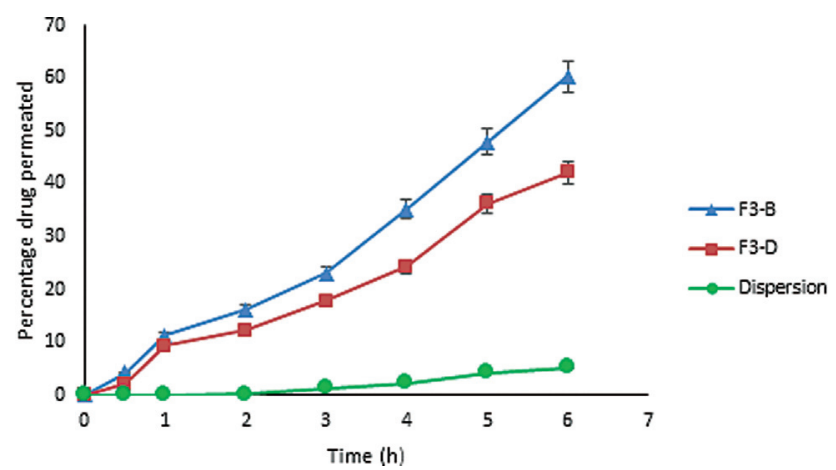

Fig. 6 Ex vivo permeation profiles of curcumin from F3-B and F3-D through Caco-2-cell monolayer. Each value is the average of three determinations with standard deviation. $p<0.05$ between $\mathrm{F} 3-\mathrm{B}$ and F3-D and $p<0.005$ between F3-B and dispersion. 
monolayer model proved that the permeation of curcumin was enhanced by piperine. The fact that piperine is a permeation enhancer of plant origin makes the oral administration of curcumin as microspheres an attractive option to the powder form administered in conventional capsules. In addition to the possibility of better bioavailability due to enhanced permeation through intestinal epithelium as confirmed by the ex vivo studies, the sustained release of curcumin allows for better therapeutic effects, reduced frequency of dosing, and good patient compliance.

\section{Conflict of Interest \\ None declared.}

\section{Acknowledgments}

The authors would like to thank the Nitte (Deemed to be University), for the use of the facilities and the funding to carry out this investigation.

\section{References}

1 Beyatricks KJ, Kumar KS, Suchitra D, Jainab NH, Anita A. Recent microsphere formulations and its applications in herbal drugs - a review. Int J Pharm Dev Technol. 2014;4(01):58-62

2 Chaturvedi M, Kumar M, Sinhal A, Saifi A. Recent development in novel drug delivery systems of herbal drugs. Int J Green Pharm. 2011;5(02):87-94

3 Sharma P, Verma S, Misri P. Global need for novel herbal drug formulations. Int J Pharmacogn Phytochem Res. 2016;8(09): 1535-1544

4 Gandhi P, Khan Z, Chakraverty N. Soluble curcumin: a promising oral supplement for health management. J Appl Pharm Sci 2011;1 (02):1-7

5 Wahlström B, Blennow GB WA study on the fate of curcumin in the rat. Acta Pharmacol Toxicol (Copenh) 1978;43(02):86-92

6 Zhang J, Tang Q, Xu X, Li N. Development and evaluation of a novel phytosome-loaded chitosan microsphere system for curcumin delivery. Int J Pharm 2013;448(01):168-174

7 Hales D, Tefas LR, Tomuță I, et al. Development of a curcuminloaded polymeric microparticulate oral drug delivery system for colon targeting by quality-by-design approach. Pharmaceutics 2020;12(11):1-24

8 Frank J, Schiborr C, Kocher A, et al. Transepithelial transport of curcumin in Caco-2 cells is significantly enhanced by micellar solubilisation. Plant Foods Hum Nutr 2017;72(01):48-53

9 Gupta T, Singh J, Kaur S, Sandhu S, Singh G, Kaur IP. Enhancing bioavailability and stability of curcumin using solid lipid nanoparticles (CLEN): a covenant for its effectiveness. Front Bioeng Biotechnol 2020;8:879

$10 \mathrm{Yu} \mathrm{H}$, Huang Q. Improving the oral bioavailability of curcumin using novel organogel-based nanoemulsions. J Agric Food Chem 2012;60(21):5373-5379

11 Zhang H, Chen B, Zhu Y, et al. Enhanced oral bioavailability of selfassembling curcumin-vitamin E prodrug-nanoparticles by conanoprecipitation with vitamin E TPGS. Drug Dev Ind Pharm 2020;46(11):1800-1808

12 Prasad C, Bhatia E, Banerjee R. Curcumin encapsulated lecithin nanoemulsions: an oral platform for ultrasound mediated spatiotemporal delivery of curcumin to the tumor. Sci Rep 2020;10 (01):8587

13 Peterson B, Weyers M, Steenekamp JH, Steyn JD, Gouws C, Hamman JH. Drug bioavailability enhancing agents of natural origin (bioenhancers) that modulate drug membrane permeation and pre-systemic metabolism. Pharmaceutics 2019;11(01):E33
14 Shoba G, Joy D, Joseph T, Majeed M, Rajendran R, Srinivas PS. Influence of piperine on the pharmacokinetics of curcumin in animals and human volunteers. Planta Med 1998;64(04): 353-356

15 Atal N, Bedi KL. Bioenhancers: revolutionary concept to market. J Ayurveda Integr Med 2010;1(02):96-99

16 Khajuria A, Thusu N, Zutshi U. Piperine modulates permeability characteristics of intestine by inducing alterations in membrane dynamics: influence on brush border membrane fluidity, ultrastructure and enzyme kinetics. Phytomedicine 2002;9(03): 224-231

17 Kesarwani K, Gupta R, Mukerjee A. Bioavailability enhancers of herbal origin: an overview. Asian Pac J Trop Biomed 2013;3(04): 253-266

18 Lengyel M, Kállai-Szabó N, Antal V, Laki AJ, Antal I. Microparticles, Microspheres, and Microcapsules for Advanced Drug Delivery. Sci Pharm 2019;87(03):20

19 Varde NK, Pack DW. Microspheres for controlled release drug delivery. Expert Opin Biol Ther 2004;4(01):35-51

20 Freiberg S, Zhu XX. Polymer microspheres for controlled drug release. Int J Pharm 2004;282(1-2):1-18

21 Sinha VR, Singla AK, Wadhawan S, et al. Chitosan microspheres as a potential carrier for drugs. Int J Pharm 2004;274(1-2):1-33

22 Moorthi C, Krishnan K, Manavalan R, Kathiresan K. Preparation and characterization of curcumin-piperine dual drug loaded nanoparticles. Asian Pac J Trop Biomed 2012;2(11):841-848

23 Baspinar Y, Üstündas M, Bayraktar O, Sezgin C. Curcumin and piperine loaded zein-chitosan nanoparticles: development and in-vitro characterisation. Saudi Pharm J 2018;26(03):323-334

24 Bolat ZB, Islek Z, Demir BN, Yilmaz EN, Sahin F, Ucisik MH. Curcumin- and piperine-loaded emulsomes as combinational treatment approach enhance the anticancer activity of curcumin on HCT116 colorectal cancer model. Front Bioeng Biotechnol 2020;8:50

25 Sareen R, Jain N, Rajkumari A, Dhar KL. pH triggered delivery of curcumin from Eudragit-coated chitosan microspheres for inflammatory bowel disease: characterization and pharmacodynamic evaluation. Drug Deliv 2016;23(01):55-62

26 Alpsten M, Bogentoft C, Ekenved G, Sölvell L. Gastric emptying and absorption of acetylsalicylic acid administered as enteric-coated micro-granules. Eur J Clin Pharmacol 1982;22(01):57-61

27 Patel KS, Patel MB. Preparation and evaluation of chitosan microspheres containing nicorandil. Int J Pharm Investig 2014;4(01):32

28 Yashaswini VK. Development of colon targeted multiparticulate drug delivery of ibuprofen. Indian J Pharm Educ Res 2013;47(02): 148-153

29 Parida P, Mishra SC, Sahoo S, Behera A, Nayak BP. Development and characterization of ethylcellulose based microsphere for sustained release of nifedipine. J Pharm Anal 2016;6(05):341-344

30 Kumar Jena G, Kumar Sahoo S, Niranjan Patra C, Charan Panigrahi K, Sahu S, Kumar Dixit P. Design, Optimization, and Evaluation of Capecitabine-loaded Chitosan Microspheres for Colon Targeting. Asian J Pharm Free full text Artic from Asian J Pharm 2017;11(03): 592

31 Badhana S, Garud N, Garud A. Colon specific drug delivery of mesalamine using eudragit S100-coated chitosan microspheres for the treatment of ulcerative colitis. Int Curr Pharm J 2013;2 (03):42-48

32 Higuchi T. Mechanism of sustained-action medication. Theoretical analysis of rate of release of solid drugs dispersed in solid matrices. J Pharm Sci 1963;52(12):1145-1149

33 Gavini E, Rassu G, Sanna V, Cossu M, Giunchedi P. Mucoadhesive microspheres for nasal administration of an antiemetic drug, metoclopramide: in-vitro/ex-vivo studies. J Pharm Pharmacol 2005;57(03):287-294

34 Rassu G, Ferraro L, Pavan B, Giunchedi P, Gavini E, Dalpiaz A. The role of combined penetration enhancers in nasal microspheres on in vivo drug bioavailability. Pharmaceutics 2018;10(04):E206 
35 Fonte P, Nogueira T, Gehm C, Ferreira D, Sarmento B. Chitosancoated solid lipid nanoparticles enhance the oral absorption of insulin. Drug Deliv Transl Res 2011;1(04):299-308

36 He P, Davis SS, Illum L. In vitro evaluation of the mucoadhesive properties of chitosan microspheres. Int J Pharm 1998;166(01): 75-88

37 Lehr CM, Bouwstra JA, Schacht EH, Junginger HE. In vitro evaluation of mucoadhesive properties of chitosan and some other natural polymers. Int J Pharm 1992;78(1-3):43-48

38 Mitra A, Dey B. Chitosan microspheres in novel drug delivery systems. Indian J Pharm Sci 2011;73(04):355-366

39 Jameela SR, Kumary TV, Lal AV, Jayakrishnan A. Progesteroneloaded chitosan microspheres: a long acting biodegradable controlled delivery system. J Control Release 1998;52(1-2):17-24
40 Peppas NA, Narasimhan B. Mathematical models in drug delivery: how modeling has shaped the way we design new drug delivery systems. J Control Release 2014;190:75-81

41 Edsman K, Hägerström H. Pharmaceutical applications of mucoadhesion for the non-oral routes. J Pharm Pharmacol 2005;57(01):3-22

42 Jameela SR, Jayakrishnan A. Glutaraldehyde cross-linked chitosan microspheres as a long acting biodegradable drug delivery vehicle: studies on the in vitro release of mitoxantrone and in vivo degradation of microspheres in rat muscle. Biomaterials 1995;16 (10):769-775. Doi: 10.1016/0142-9612(95)99639-4

43 Rohindra DR, Nand AV, Khurma JR. Swelling properties of chitosan hydrogels. South Pac J Nat Appl Sci 2004;22(01):32-35

44 Shah JC. Analysis of permeation data: evaluation of the lag time method. Int J Pharm 1993;90(02):161-169 